\title{
How Can We Improve Current Practice in Spastic Paresis?
}

\author{
Klemens Fheodoroff, ${ }^{1}$ Jorge Jacinto, ${ }^{2}$ Alexander Geurts, ${ }^{3}$ Franco Molteni, ${ }^{4}$ Jorge Hernández Franco, ${ }^{5}$ Tharaga Santiago, ${ }^{6}$ \\ Raymond Rosales ${ }^{7}$ and Jean-Michel Gracies ${ }^{6}$
}

1. Department of Neurorehabilitation Gailtal-Klinik, Hermagor, Austria; 2. Serviço de Reabilitação de Adultos 3, Centro de Medicina de Reabilitação de Alcoitão, Estoril, Portugal; 3. Radboud University Medical Centre, Nijmegen, and Sint Maartenskliniek Rehabilitation Centre, Nijmegen, The Netherlands; 4. Hospital Valduce, Villa Beretta, Costa Masnaga, Italy; 5. National Institute of Neurology and Neurosurgery M.V.S., Mexico City, Mexico; 6. Hôpitaux Universitaires Henri Mondor, Université Paris-Est, Créteil, France; 7. Royal and Pontifical University of Santo Tomas, Manila, Philippines

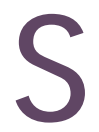

pastic paresis can arise from a variety of conditions, including stroke, spinal cord injury, multiple sclerosis, cerebral palsy, traumatic brain injury and hereditary spastic paraplegia. It is associated with muscle contracture, stiffness and pain, and can lead to segmental deformity. The positive, negative and biomechanical symptoms associated with spastic paresis can significantly affect patients' quality of life, by affecting their ability to perform normal activities. This paper - based on the content of a global spasticity interdisciplinary masterclass presented by the authors for healthcare practitioners working in the field of spastic paresis - proposes a multidisciplinary approach to care involving not only healthcare practitioners, but also the patient and their family members/carers, and improvement of the transition between specialist care and community services. The suggested treatment pathway comprises assessment of the severity of spastic paresis, early access to neurorehabilitation and physiotherapy and treatment with botulinum toxin and new technologies, where appropriate. To address the challenge of maintaining patients' motivation over the long term, tailored guided self-rehabilitation contracts can be used to set and monitor therapeutic goals. Current global consensus guidelines may have to be updated, to include a clinical care pathway related to the encompassing management of spastic paresis.

\section{Keywords}

Assessment, botulinum toxin, cerebral palsy, guided self-rehabilitation contract, hereditary spastic paraplegia, multiple sclerosis, neurorehabilitation, rehabilitation, spasticity, spinal cord injury, stroke, traumatic brain injury

Disclosure: Preparation of this paper was supported by Ipsen. The authors are responsible for the content of the paper. Ipsen did not contribute to the content of the manuscript, with the exception of a courtesy review. Klemens Fheodoroff has received unrestricted research grants from Ipsen and Merz, and honoraria for instructional courses from Ipsen, Allergan and Merz. Jorge Jacinto has received unrestricted research grants, as well as honoraria for instructional courses, conferences and advisory boards from Ipsen, Allergan and Merz. Alexander Geurts has received unrestricted research grants from Ipsen and Merz, and honoraria for instructional courses from Ipsen. Franco Molteni has received unrestricted research grants from Ipsen and Merz and honoraria for instructional courses from Ipsen, Allergan and Merz. Jorge Hernández Franco reports no conflicts of interest. Tharaga Santiago reports no conflicts of interest. Raymond Rosales has received unrestricted research grants, as well as honoraria for instructional courses, conferences and advisory boards from Ipsen and Allergan. Jean-Michel Gracies has served as a consultant and received research grant support from Allergan, I psen and Merz. This article involves a review of a meeting and did not involve any studies with human or animal subjects performed by any of the authors.

Acknowledgements: The 'Global Spasticity Masterclass' meeting was co-chaired by Professor Michael Barnes (UK) and Professor Jean-Michel Gracies (France). The authors thank all participants. Editorial assistance was provided by Vanessa Lane at MedSense Ltd., High Wycombe, UK, which was funded by Ipsen.

Authorship: All named authors meet the International Committee of Medical Journal Editors (ICMJE) criteria for authorship of this

manuscript, take responsibility for the integrity of the work as a whole, and have given final approval to the version to be published.

Open Access: This article is published under the Creative Commons Attribution Noncommercial License, which permits any non-commercial use, distribution, adaptation and reproduction provided the original author(s) and source

are given appropriate credit.

Received: 8 March 2016

Accepted: 9 May 2016

Citation: European Neurological Review, 2016;11(2):79-86

Corresponding Author: Klemens Fheodoroff, Department of Neurorehabilitation Gailtal-Klinik, Radniger Str. 12, 9620 Hermagor, Austria.

E: klemens.fheodoroff@me.com

Support: This CME meeting was supported by an unrestricted educational grant from Ipsen.
Spastic paresis may be caused by a variety of conditions, including stroke, spinal cord injury, multiple sclerosis, retroviral and other infectious spinal cord disorders, cerebral palsy, traumatic brain injury and hereditary spastic paraplegia. ${ }^{1}$ The exact prevalence of spastic paresis (in which spasticity is the most commonly recognised manifestation) is not known. However, it is estimated that around $30 \%$ of stroke survivors are affected by significant spasticity ${ }^{2}$ and $50 \%$ who present to hospital with stroke develop at least one severe contracture. ${ }^{3}$

Spastic paresis is a complex condition that may be associated with soft tissue contracture, pain and limitations of day-to-day activities, which have a substantial impact on patients' and caregivers' quality of life. ${ }^{4}$ Although treatment guidelines have been developed for (focal) spasticity, ${ }^{5}$ there remains a lack of consensus on key aspects of diagnosis, approaches to care and the care pathway that would help healthcare practitioners to more fully understand and manage this condition.

To address some of these limitations, a group of physicians and a physiotherapist with expertise in the management of spastic paresis developed a global spasticity masterclass for healthcare practitioners working in this field in order to share best practices and to discuss issues and current trends in the management of patients with spasticity. The outputs of this masterclass are presented here.

\section{Pathophysiology and definitions Spastic paresis}

Spasticity is one of several components of spastic paresis, also known as the upper motor neuron (UMN) syndrome. Spastic paresis is primarily characterised by a quantitative lack of command directed to agonist muscles involved in performing movements. ${ }^{1.6,7}$ In addition, hyperactive spinal reflexes mediate some of the positive phenomena seen in spastic paresis, while other positive symptoms are related to disordered control of voluntary movement in terms of an abnormal efferent drive or are caused 
Figure 1: Complex interactions of symptoms in spasticity

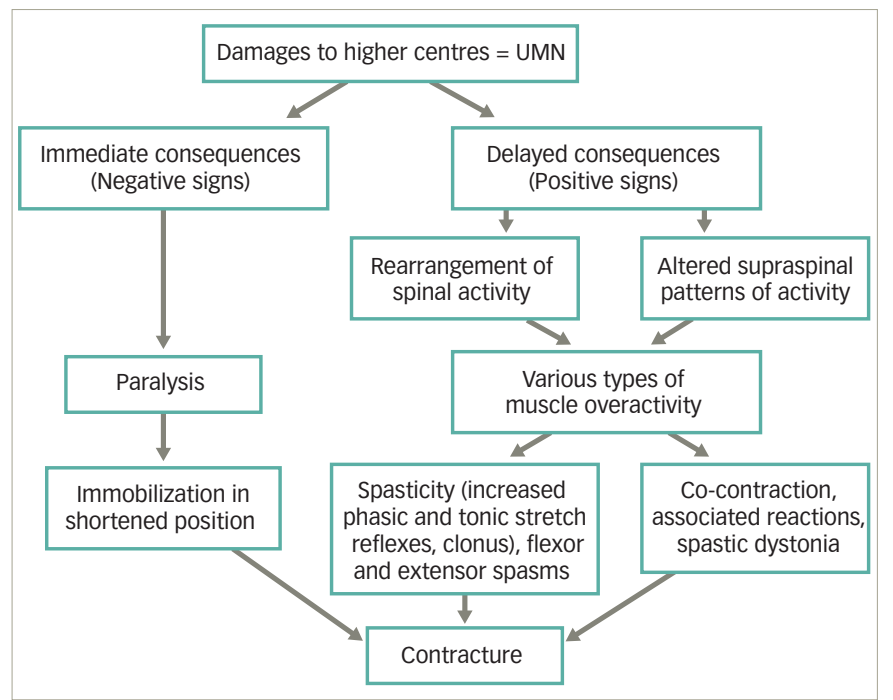

Adapted from information in Gracies ${ }^{6}$ and Gracies ${ }^{15}$; UMN = upper motor neuron

by prolonged spontaneous firing driven by so-called 'persistent inward currents' intrinsic to the motoneuron leading to 'plateau potentials'. ${ }^{8}$ A UMN lesion disturbs the balance of supraspinal inhibitory and excitatory inputs, producing a state of disinhibition of the spinal reflexes. These include proprioceptive (stretch) and nociceptive (flexor withdrawal and extensor) reflexes. The changes in muscle tone probably result from alterations in the balance of inputs from reticulospinal and other subcortical descending pathways to the motor and interneuronal circuits of the spinal cord, and the absence of an intact corticospinal system. Loss of descending tonic or phasic excitatory and inhibitory inputs to the spinal motor system and alterations in the segmental balance of excitatory and inhibitory control may be observed. ${ }^{5}$ Following brain and/ or spinal cord injury, the input from higher levels (sensorimotor cortex) may be reduced or completely lost, resulting in insufficient regulation of muscle (reflex) activity. The clinical appearance is a non-physiological flexion or extension of the affected limbs.

In spastic paresis, increased joint stiffness in the relaxed condition can be of either neural (hyperreflexia, 'spasticity') or non-neural origin (altered tissue viscoelastic properties and contractures resulting from soft tissue shortening). 19,10 Overall, three problems co-exist around each joint: stretchsensitive paresis, muscle overactivity and soft tissue contracture. ${ }^{1.6}$ Usually, spasticity precedes the occurrence of soft tissue contracture, but soft tissue shortening may also precede spasticity as a result of immobility. None of the three mechanisms of impairment are symmetrically distributed between agonist and antagonist muscles. Furthermore, reduced activity in the agonist contributes to shortening and overactivity in a usually less-paretic antagonist muscle. As a result, torque imbalance occurs around joints, which leads to the well-known limb deformities.

For more than 150 years, physiologists have tried to explain spastic paresis in terms of why and how reflexes are enhanced; however, it is now understood that the overall involvement of antagonist resistance, whether of a reflex nature or not, is the critical factor in movement impairment in spastic paresis. ${ }^{1,6,11}$

The term 'spasticity' has been inconsistently defined.12 Often, the measures used to assess spasticity do not correspond to the defined clinical features of spasticity. A definition from the 1980s focuses on spinal reflexes, describing spasticity as "a motor disorder, characterised by a velocity-dependent increase in tonic stretch reflexes (muscle tone) with exaggerated tendon jerks, resulting from hyper-excitability of the stretch reflex as one component of the UMN syndrome". ${ }^{13}$ Another definition, from the European Thematic Network to Develop Standardized Measures of Spasticity (the SPASM Consortium) in 2005, combined the positive symptoms and described spasticity as "disordered sensorimotor control, resulting from an upper motor neuron (UMN) lesion, presenting as intermittent or sustained involuntary activation of muscle". ${ }^{5}$

Existing spasticity can be worsened by other conditions such as constipation, urinary tract infections or pressure ulcers. ${ }^{14}$

\section{Signs and symptoms of spastic paresis}

Spastic paresis is characterised by a combination of positive, negative and biomechanical symptoms. Positive symptoms include tendon hyperreflexia, clonus, increased tonic stretch reflexes (hypertonia), the clasp-knife phenomenon, flexor and extensor spasms, pathological (spastic) co-contractions, associated reactions and spastic dystonia. ${ }^{6.13}$ Negative symptoms include weakness and lack of coordination. Positive and negative symptoms are primary (i.e., directly caused by the corticospinal lesion), whereas biomechanical changes are secondary; however, they all interact, resulting in muscle stiffness and contracture (Figure 1)..$^{6,15}$

\section{Spasticity}

Spasticity is the most commonly recognised manifestation of spastic paresis, but is only one of the components that characterise spastic paresis; however, it should be noted that, while nearly always present, spasticity is not always clinically detectable in patients with spastic paresis. ${ }^{16}$ Decreasing spasticity does not always improve active movement, indicating that spasticity in terms of exaggerated reflex activity may not be the main contributor to the movement impairment.

In patients with spinal cord injuries, disinhibition of spinal reflexes and prolonged spontaneous firing of spinal motoneurons may be an important mechanism associated with spasticity. In contrast, abnormal efferent activity seems to be the main mechanism in patients who have experienced a stroke and in patients with hereditary spastic paraplegia. 10,17,18 The abnormal efferent drive is often characterised by pathological (spastic) co-contraction. Spastic co-contraction is a phenomenon of supraspinal origin and is defined as an excessive degree of antagonistic activation in response to voluntary agonist command. .7.719 $^{6}$ This pathological co-contraction originates from the misdirection of the supraspinal descending pathways, particularly from the brainstem, resulting in pathologically co-activated antagonistic muscles to a point where the intended movement may be reversed in some instances. ${ }^{6}$ Stretched position of the co-contracting muscle aggravates this condition, and the extent of deformity associated with it limits the patient's movement. $6,19,20$

\section{Muscle contracture}

Muscle contracture can be the result of prolonged spasticity, but it may also precede spasticity as a result of immobility. It can begin to develop as soon as a few hours after the onset of immobilisation.

\section{Spastic dystonia}

spastic dystonia is also of supraspinal origin and is characterised by excessive tonic muscle activation at rest. It may be superimposed on soft tissue contracture, causing deformities around affected joints. ${ }^{20}$ 
These deformities have a significant impact on quality of life, and patients often desire a normalised body shape even if physical function cannot be restored.

\section{Assessment of spastic paresis}

Healthcare practitioners need to be able to assess the severity of spastic paresis to determine the most appropriate course of treatment and to monitor the progress of that treatment. A number of methods can be combined to make a full assessment.

\section{Clinical assessment}

Assessing function and mobility are the primary aim of the clinical assessment, besides recognising potential urgent issues of skin lesions or pain due to muscle overactivity. ${ }^{2}$

Verified scales provide the clinician with the means of making reproducible, routine clinical assessments of the patient's mobility and ability to perform activities of daily life. For example, active function can be assessed using the Modified Frenchay Scale, which assesses arm and hand use in 10 predefined everyday life activities, ${ }^{21}$ while a walking test (10 $\mathrm{m}$ or 2 minutes) is well validated for assessing lower limb activity.

Functional evaluation can guide further technical assessments of antagonist resistances. For such assessments, each potentia antagonistic muscle can be assessed in three validated steps:20-22

- Step 1: passive range of movement (ROM) is tested by cliniciancontrolled slow (V1) and strong movements to estimate the loss of length and level of stiffness (muscle extensibility: XV1).

- Step 2: brisk and fast passive movements (V3) are used to estimate the level of the neural reflex compon ent (partially reflecting motor neuronal excitability: XV3).

- Step 3: the active ROM against the antagonistic muscles (XA) is estimated by balancing the agonist activity against the passive and active antagonist resistances.

For each tested antagonist (e.g. biceps brachii when stretching the elbow), a coefficient of shortening can be derived (XN-XV1)/XN, where $\mathrm{XN}$ is the normal expected passive amplitude at the given joint (e.g. the elbow). A coefficient of weakness (XV1-XA)/XV1 can also be calculated, providing a sense of the motor command difficulty, taking soft tissue length and stiffness into account.

A fourth factor may also be assessed but this has not yet been validated. This is the repeatability of the agonist (e.g. triceps brachii) effort to oppose the antagonist. ${ }^{20,21}$ The patient is asked to perform rapid alternating movements of maximal amplitude against the assessed antagonist for 15 seconds and then the amplitude achieved during the last movement of the series (XA15) is measured and compared with XA. The measure of the coefficient of fatigability (XAXA15)/XA provides information on the fatigability of motor command (fatigue may potentially increase agonist paresis and antagonist cocontraction) and can be used to assess the impact of treatments.

Steps 1 and 2 above (measurement of the angles XV1 and XV3) constitute what has been termed the Tardieu Scale, ${ }^{21,22}$ and are reliable in adults and children. ${ }^{21,23}$

The widely used Ashworth Scale ${ }^{14}$ and modified Ashworth Scale ${ }^{24}$ are based on an ordinal scale that rates resistance to passive movement. The Ashworth Scale and modified Ashworth Scale may be used interchangeably. Criticisms made of the Ashworth scale are the difficulties in making comparisons between body segments and the lack of accuracy and consistency resulting from this and the need to combine measures of soft tissue contracture, spastic dystonia and spasticity to obtain a score. ${ }^{25}$

More recently, a five-step assessment has been proposed. ${ }^{21}$ This new scale incorporates the measurements of $\mathrm{XV} 1$ and $\mathrm{XV} 3$, as described in the Tardieu Scale, but also includes functional measurements such as the results of a walk test or Modified Frenchay assessment, the maximal active range of motion against the antagonist muscle and the residual active amplitude measured 15 seconds after completing a series of maximum amplitude rapid alternating movements. This expanded range of measurements provides a more accurate diagnosis of the causes of the patient's functional difficulties and allows treatment to be more precisely tailored to the patient's needs. ${ }^{21}$

\section{Clinically non-rateable phenomena}

Measurements of stretch resistance cannot discriminate between neural and non-neural mechanisms of muscle stiffness. ${ }^{26}$ Spastic cocontraction and spastic dystonia cannot be rated clinically and instead are assessed by electromyography. ${ }^{6,19}$ It should be noted, however, that this technique cannot distinguish between involuntary (reflexes) and voluntary (co-contraction) components if the limb segment is moving. However, spastic co-contraction can be easily assessed in isometric conditions. ${ }^{19}$

Spastic dystonia can be assessed at the elbow, for example, by electromyography of the elbow flexors while one passively, slowly extends the patient arm from 150 to 0 degrees; results can be compared with the activity seen in the non-paretic arm when using the same manoeuvre. ${ }^{1,6}$

Paresis may be assessed by electromyography using the twitch interpolation technique. In healthy biceps, $95-99 \%$ of motor units can be recruited during maximal voluntary effort. ${ }^{27,28}$ In patients with brain injury, the unaffected side has been shown to be approximately $10 \%$ paretic (89\% activity), and on the paretic side, around $66 \%$ of motor units were shown to be activated by motor command. 29,30

Spastic co-contraction can be assessed by asking the patient to perform isometric movements, either from a flexed or extended position. ${ }^{19}$

\section{On-going challenges in the assessment of spastic paresis}

Few studies have investigated muscle overactivity in more than a single joint and within the context of realistic, active movements. ${ }^{31}$ Another challenge when assessing spastic paresis is the fact that stretch reflexes are not unequivocally enhanced (lowered threshold, increased gain), especially during active movement. ${ }^{32}$ They are not simply velocity dependent, but are also dependent on joint position and muscle length - they are, therefore, movement dependent. ${ }^{32}$ In addition, muscle (over) activity may be present after slow stretch or even at rest (spastic dystonia). ${ }^{6}$ The ability to assess these aspects of spastic paresis remains an unmet need.

\section{Management of patients with spastic paresis}

Neurorehabilitation aims to change patients' lives to give them a higher level of independence. ${ }^{33}$ Accordingly, an improvement in the 
understanding of the required quality of neurorehabilitation has taken place in recent decades.

In a clinical setting, about one-third of patients with chronic poststroke symptoms attending rehabilitation clinics have symptomatic upper limb hypertonia; passive function (ease of care) is the domain most commonly affected, and higher Ashworth scale scores correlate with the likelihood of experiencing symptomatic muscle overactivity. ${ }^{34}$ The aim of treatment of spastic paresis is to reduce physical symptoms (including muscle tightness and spasms, fatigue and hypersensitivity to touch and pain) and improve the patient's ability to perform individual activities and participate in daily life. ${ }^{35}$ Consequently, management of a patient with spastic paresis is complex, with many factors influencing their care pathway.

Even in the early part of the 21st century, many interventions remain mostly palliative and focused on prevention of contracture, pressure sores and pain. Today, they involve significant patient participation, and diverse and targeted programmes that focus on the patient's quality of life and reintegration into society. ${ }^{36}$ Despite this, current rehabilitation treatments often have a disappointingly modest effect on impairment early or late after stroke and there are no agreed pathways for different clinical situations, although there is good evidence for use of different interventions in the upper versus lower extremities. ${ }^{37}$

The treatment approach should be tailored according to whether the patient is in the early or chronic stage of his/her condition and whether the aim is to restore or maintain function. It is important to evaluate patients on an individual level to determine the characteristics associated with their spasticity and the goals of their treatment. ${ }^{38}$

\section{Physical therapy}

Physical therapy strategies include lengthening of the shorter of the two muscles around the joint, rapid alternating movement exercises of maximal amplitude and teaching self-management strategies. ${ }^{39}$

The issue of whether stretch is effective for the treatment or prevention of contractures is debatable. A systematic review of 25 randomised controlled trials and controlled clinical trials found that regular stretch does not produce clinically important changes in joint mobility, pain, spasticity or activity limitation in people with neurological conditions. ${ }^{40}$

Nevertheless, it has been observed that stretch postures may lengthen, strengthen and widen muscles if done for an adequate daily duration. ${ }^{41}$ Lengthening the muscle is important as it can improve the range and control of movement, gait and the ability to walk up and down stairs.

The question of frequency, load and duration of muscle stretching has been addressed in a randomised controlled trial examining the efficacy of positioning the affected muscle in extension and external rotation to prevent contracture shortly after stroke..$^{41}$ Stretching activity for at least 30 minutes a day should be started as soon as possible. ${ }^{41}$ Also, asking patients to perform rapid alternating movements of maximal amplitude can help to reduce muscle co-contraction..$^{39,42-44} \mathrm{~A}$ simple exercise of repetitive finger flexion/extension movements for 15 minutes twice a day has been demonstrated to increase grip strength and peak finger extension force compared with traditional therapy. ${ }^{42}$ The benefits of repetitive exercises have also been shown for recovery of functional arm use. ${ }^{43,44}$ Finally, a programme of alternating elbow flexion/ extension movements has been shown to be effective in reducing antagonistic co-contractions and increasing voluntary control of forearm supination. ${ }^{39,45}$

Intensity can be enhanced using robotic devices from the start to increase the number of movement repetitions. Current data on available technological approaches that use high-intensity and repetitive taskspecific practice indicate that better results are achieved in the arm than the hand. ${ }^{46}$ However, in patients with long-term upper limb deficits due to stroke, robot-assisted therapy does not significantly improve motor function at 12 weeks beyond that seen with usual care or intensive therapy. ${ }^{47}$ Similar results have been seen in lower limb rehabilitation. ${ }^{48}$ If muscle overactivity occurs, injection with botulinum neurotoxin (BoNT; see below) may decrease co-contraction and will allow the patient to continue with intensive rehabilitation. ${ }^{49}$ This approach contributes to brain plasticity more effectively in the early stages of recovery, leading to enhanced recovery of voluntary control of segmental limb movements. ${ }^{50}$

Bimanual rehabilitation is also used in patients after stroke.51 Bimanual co-ordination in patients during standard post-stroke rehabilitation starts to become efficient 6 weeks after onset of stroke, and so it is recommended after this time point. ${ }^{51}$ A systematic review on current neurorehabilitation therapies for spasticity attests to the robustness of some techniques over others. ${ }^{52}$

\section{Botulinum toxin A}

Botulinum toxin type $\mathrm{A}$ (BoNT-A) has a grade $\mathrm{A}$ recommendation from the American Academy of Neurology for the treatment of spasticity in adults and children. ${ }^{53,54} \mathrm{~A}$ meta-analysis of 16 clinical studies with BoNT-A indicated that it safely and effectively decreases muscle tone and increases range of motion. ${ }^{55}$

BONT-A has dual peripheral effects, namely the chemodenervation of cholinergic transmission in both extrafusal and intrafusal muscle fibres. ${ }^{56}$ BoNT-A-induced changes in sensory input through denervation of the intrafusal muscle fibres may be essential in modulating loss of presynaptic inhibition in dystonia. In addition, BoNT-A injections into the shorter of the two co-contracting muscles around the joint will augment stretching activities. There is also some evidence for modulation of sensorimotor loops at the spinal and supraspinal level, as assessed by neurophysiological and functional magnetic resonance imaging (MRI) technologies (i.e. before and after BoNT-A treatment). ${ }^{57}$

BoNT-A requires 24-72 hours to take effect. The peak effect occurs from 10 days to 4 weeks, depending on the clinical situation; clinical improvements usually last for up to 12 to 20 weeks. ${ }^{58}$ It must be recognised that BoNT-A treatment is limited in widespread multifocal muscle overactivity, and treatment effects are only temporary, ${ }^{20,51}$ opening a therapeutic window for a combined neurorehabilitative approach. The reversibility of BoNT-A effects may lead to repeated treatment in chronic muscle overactivity, but may perhaps modify the course of muscle overactivity in early post-stroke intervention. ${ }^{59}$

Several studies have investigated the effect of BoNT-A on post-stroke upper limb function and mobility, usually combined with an exercise programme ${ }^{43,60-63}$ Treatment with BoNT-A gave a notable improvement in hand function, and hence improvements in self-care tasks and other activities of daily living, and alleviated pain. Reaching and grasping functions were improved but not significantly so. These findings suggest that - in contrast to the traditional approach - it may be worth initially focusing on recovery of control of hand movements after stroke. 
The optimal time to administer BoNT-A may be when muscle overactivity becomes evident and bothersome to the patient, resulting in impairment of active and passive functions, disability and associated reactions, or when it induces pain. ${ }^{59}$ The Upper Limb International Spasticity Study (ULIS)-II showed that the large majority (80\%) of patients with post-stroke upper limb spasticity treated with BoNT-A in a real-life clinical setting achieved their treatment goals, mainly in terms of passive and active functions and pain reduction. ${ }^{64}$

Early single-dose BoNT-A treatment ( $<3$ months after stroke) of spasticity has been investigated in three upper limb studies (summarised in Figure 2). ${ }^{65-68}$ In the first study, a small cohort of patients with no arm function was found to benefit functionally. ${ }^{65}$ In the second study, development of disabling finger flexion was significantly reduced compared with placebo. ${ }^{66}$ In the third study, a sustained reduction in muscle tone was observed for 6 months following a single fixed dose of BoNT-A. ${ }^{67}$ Early use of BoNT-A may extend the time window for motor re-learning with physiotherapy by decreasing overactive extrafusal muscle fibres and reducing muscle spindle sensitivity through chemodenervation of intrafusal muscle fibres. ${ }^{69}$ In effect, the early BoNT-A intervention paradigm may potentially modify the natural progress of spasticity, prevent spasticity/dystoniarelated complications or even delay re-injection. Nevertheless, it is important to consider that there may be a time lag between maximum reduction in tone and functional gain, possibly related to the time required to learn how to use the increased segmental ROM after reduction in hypertonicity. ${ }^{63}$

Clinical trials of BONT-A use in infants and children with spasticity due to cerebral palsy have demonstrated that contractures and deformities during growth can be reduced, spasticity in the upper and lower extremities reduced and ROM in the joints of the lower limbs increased. ${ }^{70-72}$ Early use of BoNT-A in key muscles in children with cerebral palsy seems to limit the development of spasticity and to increase their likelihood of achieving important developmental milestones (e.g. sitting, crawling and standing). ${ }^{73}$

A systematic review of methods to improve outcomes following BoNT-A for treatment of limb spasticity identified 10 adjunct therapies. ${ }^{74}$ There was level 1 evidence for electrical stimulation, modified constraintinduced movement therapy and physiotherapy and level 2 evidence for casting and dynamic splinting. ${ }^{74}$ In contrast, there was level 1 and 2 evidence that adjunct taping, segmental muscle vibration, cyclic functional electrical stimulation and motorised arm ergometer may not improve outcomes compared with BoNT-A injections alone. ${ }^{74}$

\section{Other pharmacological interventions}

A systematic review of the effectiveness of pharmacological interventions in reducing spasticity of the lower limb in chronic stroke survivors analysed outcomes from nine randomised controlled trials. ${ }^{75}$ Of these, four provided evidence of BoNT-A efficacy. ${ }^{75}$ One study provided evidence that both alcohol and phenol neurolytics were effective in reducing spasticity ${ }^{75}$ The final four studies provided evidence that oral and intrathecal medications were effective in reducing lower limb spasticity compared to placebo. ${ }^{75}$

\section{Assistive devices}

Assistive devices can help with lost function and provide the patient with a more independent way of living. They should not, however, be used as substitutes for physiotherapy. The cost of such devices does not have to be high to be of benefit to the patient (e.g. simple
Figure 2: The role of BoNT-A therapy in early intervention after stroke

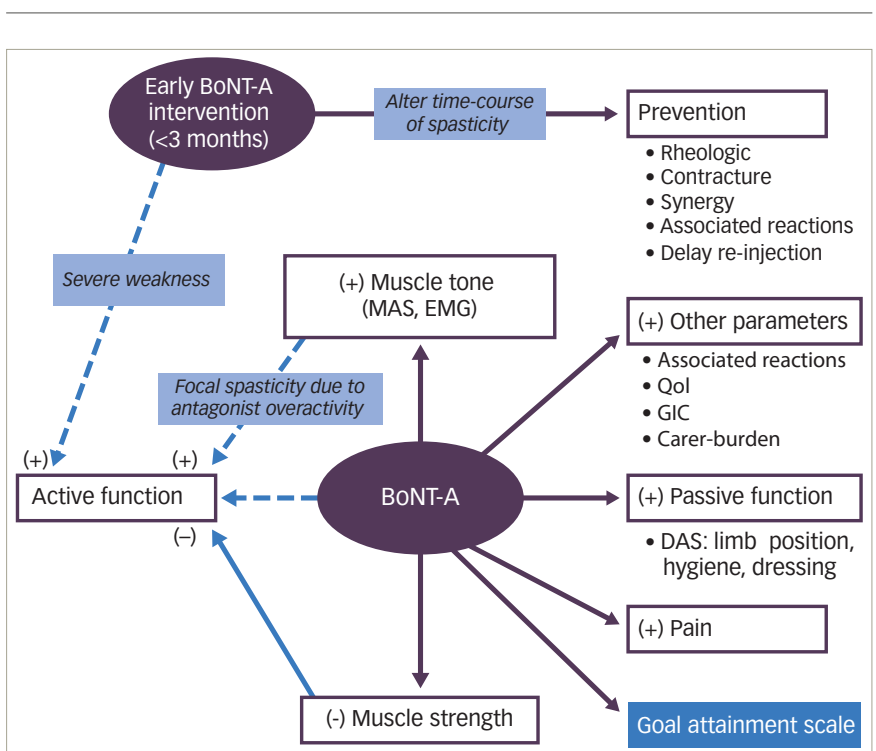

Incorporates information from cousins et al, Hesse et al and Rosales et a| ${ }^{65-67}$ and is adapted with permission from Rosales et a $1{ }^{88}$ BONT-A = botulinum toxin A; $D A S=$ Disability Assessment Scale; $E M G$ = Electromyography; GIC = Global Impression of Change; MAS = Modified Ashworth Scale; QOL = Quality of life

adaptation of a wheelchair for one-handed control). Low budget assistive devices can also be used to aid rehabilitation exercises, such as the resonating arm exerciser. This simple mechanical device, which snaps onto a manual wheelchair, uses resonance to assist in arm training, and provides an effective method for increasing arm movement ability in patients after severe chronic hemiparetic stroke. ${ }^{76}$

\section{Future Options for Treatment}

The ipsilateral motor pathway is a normal motor control pathway and is important clinically because it can contribute to motor recovery in the early stages after the onset of stroke. ${ }^{77}$ The pathway innervates proximal muscles and contributes more to movements of the legs than of the hands.

Virtual reality rehabilitation-based therapy in post-stroke patients has been investigated. In a recently published study, motor rehabilitation was promoted by challenging patients with simple computer games representative of daily activities of self-support. ${ }^{78}$ This therapy has demonstrated clinical value, although the underlying changes in neuronal reorganisation responsible for the behavioural improvements are unclear. The use of new technologies, such as robotic and noninvasive brain stimulation for neurorehabilitation, may also be appropriate for both in- and outpatient rehabilitation in the future. Tests using functional MRI have shown that contralesional activation of the unaffected motor cortex, cerebellar recruitment and compensatory prefrontal cortex activation were the most prominent activations involved with movement command. ${ }^{78}$ Upon commencing therapy there was a significant correlation between motor dexterity and total recruited activity $(p<0.05)$, and overall brain activity during therapy was inversely related to normalised behavioural improvements $(p<0.05) .^{78}$ It would appear, therefore, that prefrontal cortex and cerebellar activity are the driving forces of the recovery associated with this type of therapy.

\section{The care pathway in spastic paresis}

Patients with acquired brain injuries can be challenged when coming to terms with their disability. If their high expectations for recovery are not 
Figure 3: Community reintegration after stroke

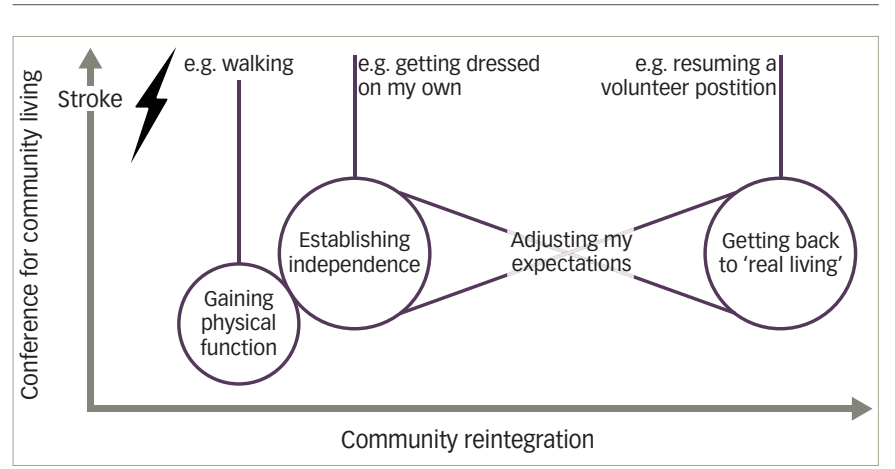

Figure 4: Transitioning care via a multidisciplinary team approach in patients with acquired brain injury

\begin{abstract}
Masterclass recommendations
The journey for a patient with an acquired brain injury begins in the intensive care unit, where the immediate priority is the life of the patient. After moving to the acute rehabilitation care unit, the second priority is preservation of muscle length. Family and carers will be integrated into the partnership with the rehabilitation team before the patient's transition through the rehabilitation centre and back into daily life. Effective and smooth transitioning from acute to long-term management requires clear communication pathways between all stakeholders.

Patients should be carefully evaluated and frequently reviewed in team meetings, and the timing and structure of discharge should be planned around the individual patient's needs. This will include planning for home visits, interaction with the community to prepare for housing, continued support on an outpatient basis and - in some cases - return to work or school. A comprehensive, standardised and easy to understand discharge report, which includes instructions for self-stretch, active self-exercises and a follow-up plan, should be provided to the primary care team.
\end{abstract}

met, they may enter a period of mourning or despair and/or develop a loss of self-worth and feelings of isolation. ${ }^{79}$ Patients then either reject the need for further help or develop a more positive attitude of determination to beat the new obstacle facing them. If guided carefully, patients will ultimately adjust towards accepting the disability as a new part of themselves, learning to cope with it and integrating it into their daily lives.

Community reintegration after acquired brain injury involves many stages (Figure 3), ${ }^{80}$ beginning with regaining physical function and establishing independence, both of which start within the clinical setting. After discharge, patients then need to adjust their expectations to what they can do in their home environment. They will often have a lot of free time as a result of the impact of the stroke on their ability to carry out their usual daily routine, and they need to learn to fill this gap with meaningful and achievable activities. Here, self-management approaches will be essential to overcome a threatening halt in rehabilitation activities. ${ }^{45}$

Individual coping strategies after a stroke may influence recovery. A review of 14 studies reporting ten different types of coping strategies found that there was considerable stability in coping strategies over the long term, whether they be problem-focused, emotion-focused or avoidance strategies. ${ }^{81}$ Patients demonstrating avoidance coping strategies need particular attention because they are at increased risk of anxiety and depression.

\section{The multidisciplinary approach}

A multidisciplinary approach to the care of patients with spastic paresis, with clear and effective communication between all stakeholders (including the patient and his/her family), may improve outcomes. Recent evidence supports a multidisciplinary approach, involving not only the healthcare team (clinician[s], physical therapist, occupational therapist, etc.), but also the patient and their family or caregiver(s). ${ }^{35,80}$ However, more evidence is needed to establish the optimal types (modalities, therapy approaches and settings) and intensities of therapy for improving active and passive function in adults and children with post-stroke spasticity, in the short and longer term. ${ }^{82}$

The recommendations for a multidisciplinary approach are summarised in Figure 4.

\section{Role of the physician}

From the clinical perspective, a number of obstacles may need to be addressed, such as lack of agreement regarding level of functioning and agreed care pathways, when to begin treatment and the goals to be set for the long term. Many physicians have limited time to set goals and analyse outcomes. Improved training of physicians and the use of a standardised list are key in improving goal-setting in clinical practice. A number of factors will influence and are relevant to neurorehabilitation and should be taken into account. These include: patient-related factors, e.g. state of mind and personality; exercise-related factors, e.g. type, intensity and structure; physician-related factors, e.g. the ability to give adequate feedback; and environmental impact. ${ }^{83}$

The ULIS programme has helped to identify what physicians say they do (ULIS-I) and what they really do (ULIS-II) in routine clinical practice. ${ }^{64}$

\section{Role of the physical and occupational therapist}

Physical and occupational therapy are key interventions within multidisciplinary care, not only to prevent orthopaedic complications as a result of muscle shortening, ${ }^{41}$ but also to establish an adequate exercise programme and set realistic goals. It is vital that the patient commits to performing individual daily exercises and documenting them regularly, so that the therapists can review them. This allows feedback on current and expected performance, as well as technical and psychological support. This would be a key component of a guided self-rehabilitation contract (see below) between patients and their clinical teams, including physical and occupational therapists. ${ }^{45}$

\section{Role of family/caregivers}

Involving the patient's family and/or caregivers with the rehabilitation team from the early stages of neurorehabilitation through to longterm follow-up is known to work well. ${ }^{84}$ Family and caregivers are key members of the team and it is important that they receive all necessary information and support from the beginning of the treatment journey. They should be aware of the goals of therapy and what their role will be. Their concerns regarding impairments and symptoms experienced by the patient should be addressed and they should also be encouraged to share relevant details that could inform treatment. This will help to promote confidence in managing the patient's needs. An important step is to implement home visits prior to hospital discharge. Realistic goals should take into account the individual's capacity to follow instructions, 
as well as patient, family and caregiver opinions on the feasibility of tasks. This approach helps caregivers to improve their knowledge of the patient's condition, creates realistic expectations of the benefits and harms of chosen options and reduces potential conflicts and passivity in decision-making.

\section{Guided self-rehabilitation contracts}

Behavioural experience is fundamental to the promotion of brain plasticity. After a brain lesion has occurred, patients often present with self-imposed hypoactivity, which can cause a halt in brain plasticity and might contribute to worsening capacities of neuronal recruitment. ${ }^{85}$ Ultimately, this may lead to additional cognitive and motor decline, which in turn further promotes hypoactivity and becomes a vicious cycle of 'disuse'. The process of rehabilitation after a stroke needs a long-term commitment and requires dedicated time every day in order to improve outcomes and escape the potentially vicious cycle. ${ }^{45,86-88}$ In most cases, more than an hour of rehabilitation is needed daily.

The challenge is to motivate patients to adhere to a long-term selfrehabilitation programme of repetitive and difficult exercises. This motivation can be achieved through appropriate goal-setting, technical guidance (guided self-rehabilitation) and psychological support, mediated by regular and long-standing follow-up by a rehabilitation professional. All of these actions will help patients to achieve and maintain the state of mind necessary to acquire new motor skills. .33,45 $^{33}$

As part of a guided self-rehabilitation contract, it has been proposed that patients agree to undertake and document a series of individualised daily exercises, which are as important as taking daily medication, and the therapist agrees to coach the patient (technical guidance and encouragement) over the long term. The terms of the contract are based on the examination by the clinician and therapists, and entail an acceptance that rehabilitation is a long-term undertaking. The contract can be updated as the patient progresses. A 2-year, multicentre, randomised controlled trial (Neurorestore) is currently underway to compare the value of guided self-rehabilitation contracts with that of conventional community therapy, in terms of functional, psychological and social impact (clinicaltrials.gov identifier: NCT02202954). Potential benefits of this process are expected to include optimisation of the output of individual physiotherapy sessions; increased daily duration of active exercise performed by the patient and the provision of on-going counselling and care over the long term. It is anticipated that limitations may arise when patients have severe cognitive impairments, such as major aphasia or difficulties with attention or comprehension. The success of this approach will also depend on the commitment of the patient's family and/or caregiver

\section{Summary}

This review of some of the key aspects of the assessment and treatment of patients with spastic paresis highlights the need for a cohesive, multidisciplinary approach to the patient's journey from diagnosis of acquired brain injury to long-term rehabilitation.

Approaches to this should include the setting and monitoring of realistic therapeutic goals, in particular through the use of guided self-rehabilitation contracts, which could help provide more intensive rehabilitation to patients. Treatment plans should also consider therapeutic agents, where appropriate. Improved training is needed for healthcare practitioners to raise awareness of the pathophysiology of spastic paresis and hence the value of integrated treatment plans that include rehabilitation therapy in the acute phase. $]$

\section{CALL TO ACTION}

There is an outstanding need to optimise the patient journey and treatment outcomes in spastic paresis. To accomplish these goals, we must:

- Educate: not only clinicians and physiotherapists, but also patients' family members and caregivers, and, vitally, the patients themselves

- Communicate: between the stakeholders so that all are aware of the patient's treatment goals and progress, and of new treatment opportunities

- Integrate: between specialist rehabilitation units and community centres and using a multidisciplinary team approach comprising clinicians, physiotherapists, caregivers and patients

- Promote: personalised goal-setting in clinical practice to engage patients

- Facilitate: the use of individualised guided self-rehabilitation contracts, once they are proven to be more effective than conventional therapy

- Innovate: with continued research into assessment and therapies for spastic paresis
1. Gracies J-M, Pathophysiology of spastic paresis. I: paresis and soft tissue changes, Muscle Nerve, 2005;31:535-51.

2. Watkins CL, Leathley MJ, Gregson JM, et al., Prevalence of spasticity post stroke, Clin Rehabil, 2002:16:515-22.

3. Kwah LK, Harvey LA, Diong JH, et al., Half of the adults who present to hospital with stroke develop at least one contracture within six months: an observational study, J Physiother, 2012:58:41-7.

4. Zorowitz RD, Gillard PJ, Brainin M, Poststroke spasticity: sequelae and burden on stroke survivors and caregivers, Neurology, 2013;80(suppl 2):S45-S52.

5. Centre for Rehabilitation and Engineering Studies (CREST), SPASM. A European Thematic Network to Develop Standardised Measures of Spasticity. CREST, University of Newcastle; 2014. Available from: http://research ncl ac uk/spasm/Projdescrhtm (accessed 11 November 2015)

6. Gracies J-M, Pathophysiology of spastic paresis. II: emergence of muscle overactivity, Muscle Nerve, 2005;31:552-71.

7. Sheean $\mathrm{G}$, The pathophysiology of spasticity, Eur J Neurol, 2002;9(suppl 1):3-9, 53-61

8. Gorassini MA, Knash ME, Harvey PJ, et al., Role of motoneurons in the generation of muscle spasms after spinal cord injury, Brain, 2004;127:2247-58.

9. Olsson MC, Krüger M, Meyer L-H, et al., Fibre type-specific increase in passive muscle tension in spinal cord-injured subjects with spasticity, J Physiol, 2006;577:339-52.

10. Lieber RL, Steinman S, Barash IA, et al., Structural and functional changes in spastic skeletal muscle, Muscle Nerve, 2004;29:615-27.

11. Ada L, Vattanasilp W, O'Dwyer NJ, et al., Does spasticity contribute to walking dysfunction after stroke? I Neurol Neurosurg PSychiatry, 1998;64:628-35.

12. Malhotra S, Pandyan AD, Day CR, et al., Spasticity, an impairment that is poorly defined and poorly measured, Clin Rehabil, 2009:23:651-58.

13. Lance JW, The control of muscle tone, reflexes, and movement Robert Wartenberg Lecture, Neurology, 1980;30:1303-13.

14. Kheder A, Padmakumari K, Nair S, Spasticity: pathophysiology, evaluation and management, Pract Neurol, 2012;12:289-98

15. Gracies JM, Pathophysiology of impairment in patients with spasticity and use of stretch as a treatment of spastic with spasticity and use of stretch as a treatment o: 5pastic

16. Ashworth B, Preliminary trial of carisoprodol in multiple sclerosis, Practitioner, 1964;192:540-2.

17. de Niet M, Latour H, Hendricks $H$, et al., Short-latency stretch reflexes do not contribute to premature calf muscle activity during the stance phase of gait in spastic patients, Arch Phys Med Rehabil, 2011;92:1833-9.

18. Burke D, Wissel J, Donnan GA, Pathophysiology of spasticity in stroke, Neurology, 2013;80(suppl 2):S20-S26.

19. Vinti $\mathrm{M}$, Couillandre $\mathrm{A}$, Hausselle J, et al., Influence of effort intensity and gastrocnemius stretch on co-contraction and torque production in the healthy and paretic ankle, Clin Neurophysiol, 2013;124:528-35.
20. Gracies $J-M$, Coefficients of impairment in deforming spastic paresis, Ann Phys Rehabil Med, 2015:58:173-8.

21. Gracies J-M, Bayle N, Vinti M, et al., Five-step clinica assessment in spastic paresis, Eur J Phys Rehabil Med 2010:46:411-21

22. Gracies JM, Marosszeky JE, Renton R, et al., Short-term effects of dynamic lycra splints on upper limb in hemiplegic patients. Arch Phys Med Rehabil, 2000;81:1547-55.

23. Ben-Shabat E, Palit M, Fini NA, et al., Intra- and interrater reliability of the Modified Tardieu Scale for the assessment of lower limb spasticity in adults with neurologic injuries, Arch Phys Med Rehabil, 2013;94:2494-501.

24. Bohannon RW, Smith MB, Interrater reliability of a modified Ashworth scale of muscle spasticity. Phys Ther, 1987:67:206-7.

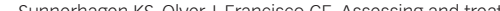
funn functional impairment in postst

26. Aloraini SM, Gäverth J, Yeung E, et al., Assessment of spasticity after stroke using clinical measures: a systematic review, Disabil Rehabil, 2015;37:1-11.

27. Allen GM, McKenzie DK, Gandevia SC., Twitch interpolation of the elbow flexor muscles at high forces, Muscle Nerve, 1998;21:318-28.

28. Gandevia SC, Allen GM, Butler JE, et al., Supraspinal factors in human muscle fatigue: evidence for suboptimal output from the motor cortex, J Physiol, 1996;490(Pt 2):529-36.

29. Newham DJ, Hsiao SF, Knee muscle isometric strength, 
voluntary activation and antagonist co-contraction in the first six months after stroke, Disabil Rehabil, 2001;23:379-86.

30. Riley NA, Bilodeau $M$, Changes in upper limb joint torque patterns and EMG signals with fatigue following a stroke, Disabil Rehabil, 2002:24:961-9.

31. Musampa NK, Mathieu PA, Levin MF, Relationship between stretch reflex thresholds and voluntary arm muscle activation in patients with spasticity, Exp Brain Res, 2007:181:579-93.

32. Trompetto C, Marinelli L, Mori L, et al., Pathophysiology of spasticity: implications for neurorehabilitation, Biomed Res Int, 2014;2014:354906.

33. United Nations, Standard rules on the equalization of opportunities for persons with disabilities. United Nations 1994 Available from: www.un.org/disabilities/default.asp?id=26 (accessed 11 November 2015)

34. Kong K-H, Chua KS-G, Lee J, Symptomatic upper limb spasticity in patients with chronic stroke attending a rehabilitation clinic: frequency, clinical correlates and predictors, J Rehabil Med, 2010:42:453-7.

35. Ashford S, Fheodoroff $\mathrm{K}$, Jacinto J, et al., Common goal areas in the treatment of upper limb spasticity: A multicentre analysis, Clin Rehabil, 2015:[Epub ahead of print].

36. Rentsch HP, Bucher PO, Tesak J, [ICF in rehabilitation: the practical application of the international classification of functioning, disability and health in rehabilitation everyday] In German, Idstein: Schulz-Kirchner Verlag GmbH; 2005.

37. Foley N, Mehta S, Jutai J, et al., Upper extremity interventions. In: Teasell $\mathrm{R}$, ed. Evidence-based review of stroke rehabilitation. London, Ontario: Sockit Solutions; 2013:1-163:chap 10. Available from: www.ebrsr.com/evidence-review/10-uppe extremity-interventions (accessed 12 November 2015).

38. Sheean $G$, Lannin NA, Turner-Stokes $L$, et al., Cerebral Pals Institute. Botulinum toxin assessment, intervention and after-care for upper limb hypertonicity in adults: internationa consensus statement, Eur J Neurol, 2010;17(suppl 2):74-93.

39. Hu $X$, Tong $K Y$, Song $R$, et al., Variation of muscle coactivation patterns in chronic stroke during robot-assisted elbow training Arch Phys Med Rehabil, 2007:88:1022-9.

40. Katalinic OM, Harvey LA, Herbert RD, Effectiveness of stretch for the treatment and prevention of contractures in people with neurological conditions: a systematic review, Phys Ther, 2011;91:11-24.

41. Ada L, Goddard E, McCully J, et al., Thirty minutes of positioning reduces the development of shoulder external rotation contracture after stroke: a randomized controlled trial, Arch Phys Med Rehabil, 2005;86:230-4.

42. Bütefisch C, Hummelsheim H, Denzler P, et al., Repetitive training of isolated movements improves the outcome of motor rehabilitation of the centrally paretic hand, I Neurol SCi, 1995;130:59-68.

43. Feys $H M$, De Weerdt WJ, Selz BE, et al., Effect of a therapeutic intervention for the hemiplegic upper limb in the acute phase after stroke: a single-blind, randomized, controlled multicenter trial, Stroke, 1998;29:785-92.

44. Feys H, De Weerdt W, Verbeke G, et al., Early and repetitive stimulation of the arm can substantially improve the long-term outcome after stroke: a 5-year follow-up study of a randomize trial, Stroke, 2004;35:924-9.

45. Gracies JM, Blondel R, Gault-Colas C, et al., [Guided self-rehabilitation contract in spastic paresis] In French, (c)Association Neuroloco, Paris 2013, 108p, ISBN 978-2-35327$169-6$

46. Langhorne P, Coupar F, Pollock A, Motor recovery after stroke: a systematic review, Lancet Neurol, 2009;8:741-54.

47. Lo AC, Guarino PD, Richards LG, et al., Robot-assisted therapy for long-term upper-limb impairment after stroke, N Eng/ I Med, 2010;362:1772-83.

48. Westlake KP, Patten C, Pilot study of Lokomat versus manualassisted treadmill training for locomotor recovery post-stroke, I Neuroeng Rehabil, 2009:6:18.

49. Langhorne P, Bernhardt J, Kwakkel G, Stroke rehabilitation,
Lancet, 2011;377:1693-702.

50. Dipietro L, Krebs HI, Fasoli SE, et al., Submovement changes characterize generalization of motor recovery after stroke, Cortex, 2009:45:318-24.

51. Metrot J, Mottet $D$, Hauret I, et al., Changes in bimanua coordination during the first 6 weeks after moderate hemiparetic stroke, Neurorehabil Neural Repair, 2013:27:251-9.

52. Veerbeek JM, van Wegen $E$, van Peppen $R$, et al., What is the evidence for physical therapy poststroke? A systematic review and meta-analysis, PLoS One, 2014;9:e87987.

53. Simpson DM, Gracies JM, Graham HK, et al., Therapeutics and Technology Assessment Subcommittee of the American Academy of Neurology. Assessment: Botulinum neurotoxin for the treatment of spasticity (an evidence-based review): report of the Therapeutics and Technology Assessment Subcommittee of the American Academy of Neurology, Neurology 2008;70:1691-8.

54. Gracies JM, Bayle N, Goldberg S, et al., Botulinum toxin type B in the spastic arm: a randomized, double-blind, placebocontrolled, preliminary study, Arch Phys Med Rehabi 2014;95:1303-11

55. Ozcakir S, Sivrioglu K, Botulinum toxin in poststroke spasticity, Clin Med Res, 2007;5:132-8.

56. Rosales RL, Dressler D, On muscle spindles, dystonia and botulinum toxin, Eur J Neurol, 2010;17(suppl 1):71-80.

57. Caleo $\mathrm{M}$, Antonucci F, Restani $\mathrm{L}$, et al. A reappraisal of the central effects of botulinum neurotoxin type $A$ : by what central effects of botulinum neurotoxin type A:
mechanism? I Neurochem, 2009:109:15-24.

58. Nigam PK, Nigam A, Botulinum toxin, Indian J Dermatol, 2010;55:8-14.

59. Rosales RL, Dystonia, spasticity and botulinum toxin therapy: rationale, evidences and clinical context. In Rosales RL, ed. Dystonia - many facets. Rijeka, Croatia: InTech; 2012:83-98. Available from: www.intechopen.com/books/dystonia-themany-facets. (accessed 11 November 2015).

60. Shaw LC, Price $\mathrm{Cl}$, van Wijck FM, et al., BoTULS Investigators. Botulinum Toxin for the Upper Limb after Stroke (BOTULS) Trial: effect on impairment, activity limitation, and pain, Stroke, 2011;42:1371-79.

61. Diserens K, Ruegg D, Kleiser R, et al., Effect of repetitive arm cycling following botulinum toxin injection for poststroke spasticity: evidence from FMRI, Neurorehabil Neural Repair 2010;24:753-62

62. Veverka T, Hluštík P, Tomášová Z, et al., BoNT-A related changes of cortical activity in patients suffering from severe hand paralysis with arm spasticity following ischemic stroke J Neurol Sci, 2012;319:89-95

63. Francis HP, Wade DT, Turner-Stokes $L$, et al., Does reducing spasticity translate into functional benefit? An exploratory meta-analysis, J Neurol Neurosurg Psychiatry, 2004:75:1547-51.

64. Turner-Stokes L, Fheodoroff K, Jacinto J, et al., Results from the Upper Limb International Spasticity Study-II (ULISII):a large international, prospective cohort study investigating practice and goal attainment following treatment with botulinum toxin in real-life clinical management, BMJ Open, 2013;3:e002771.

65. Cousins E, Ward A, Roffe C, et al., Does low-dose botulinum toxin help the recovery of arm function when given early after stroke? A phase II randomized controlled pilot study to estimate effect size, Clin Rehabil, 2010:24:501-13.

66. Hesse S, Mach H, Fröhlich S, et al., An early botulinum toxin A treatment in subacute stroke patients may prevent a disabling finger flexor stiffness six months later: a randomized controlled trial, Clin Rehabil, 2012;26:237-45.

67. Rosales RL, Kong KH, Goh KJ, et al., Botulinum toxin injection for hypertonicity of the upper extremity within 12 weeks after stroke: a randomized controlled trial, Neurorehabil Neural Repair, 2012;26:812-21.

68. Rosales RL, Dystonia, spasticity and botulinum toxin therapy: rationale, evidences and clinical context, dystonia - the many facets. 2012 Prof. Raymond Rosales (ed), ISBN: 978-95351-0329-5, InTech, DOl: http://dx doi.org/10.5772/27435.
Available from: http://www.intechopen.com/books/dystonia the-many-facets/dystonia-spasticity-and-botulinum-toxintherapy-rationale-evidences-and-clinical-context (accessed 12 December 2015).

69. Rosales RL, Kanovsky P, Fernandez HH, What's the "catch" in upper-limb post-stroke spasticity: expanding the role of botulinum toxin applications, Parkinsonism Relat Disord, 2011:17(suppl 1):S3-S10

70. Pascual-Pascual SI, [Use of botulinum toxin in the preventive and palliative treatment of the hips in children with infantile cerebral palsy], Rev Neurol, 2003;37:80-2.

71. Pascual-Pascual SI, Pascual-Castroviejo I, Safety of botulinum toxin type A in children younger than 2 years, Eur J Paediatr Neurol, 2009;13:511-15.

72. Druschel C, Althuizes HC, Funk JF, et al., Off label use of botulinum toxin in children under two years of age: a systematic review, Toxins (Basel), 2013:5:60-72.

73. Placzek R, Siebold D, Funk JF, Development of treatment concepts for the use of botulinum toxin a in children with cerebral palsy, Toxins (Basel), 2010;2:2258-71.

74. Mills PB, Finlayson H, Sudol M, O'Connor R, Systematic review of adjunct therapies to improve outcomes following botulinum toxin injection for treatment of limb spasticity, Clin Rehabil, 2015; pii: 0269215515593783. [Epub ahead of print]

75. McIntyre A, Lee T, Janzen S, et al., Systematic review of the effectiveness of pharmacological interventions in the treatment of spasticity of the hemiparetic lower extremity more than six months post stroke, Top Stroke Rehabil, 2012;19:479-90.

76. Zondervan DK, Palafox L, Hernandez J, et al., The Resonating Arm Exerciser: design and pilot testing of a mechanically passive rehabilitation device that mimics robotic activ assistance, J Neuroeng Rehabil, 2013;10:39.

77. Jang $\mathrm{SH}, \mathrm{A}$ review of the ipsilateral motor pathway as a recovery mechanism in patients with stroke, NeuroRehabilitation, 2009;24:315-20.

78. Orihuela-Espina F, Fernández del Castillo I, Palafox L, et al., Neural reorganization accompanying upper limb motor rehabilitation from stroke with virtual reality-based gesture therapy, Top Stroke Rehabil, 2013:20:197-209.

79. Bhimani RH, MCAlpine $\mathrm{CP}$, Henly SJ, Understanding spasticity from patients' perspectives over time, J Adv Nurs, 2012;68:2504-14.

80. Wood JP, Connelly DM, Maly MR, 'Getting back to real living': A qualitative study of the process of community reintegration after stroke, Clin Rehabil, 2010;24:1045-56.

81. Donnellan C, Hevey D, Hickey A, et al., Defining and quantifying coping strategies after stroke: a review. J Neurol Neurosurg Psychiatry, 2006:77:1208-18.

82. Demetrios $M$, Khan F, Turner-Stokes $L$, et al., Multidisciplinary rehabilitation following botulinum toxin and other focal intramuscular treatment for post-stroke spasticity, Cochrane Database Syst Rev, 2013;6:CD009689.

83. Van Cranenburgh $B$, Neurorehabilitation: neurophysiologisch Grundlagen, Lernprozesse, Behandlungsprinzipien. Munich: Elsevier GmbH, Urban \& Fischer Verlag; 2007.

84. Hartmann M, Bäzner E, Wild B, et al., Effects of interventions involving the family in the treatment of adult patients with chronic physical diseases: a meta-analysis, PSychother Psychosom, 2010;79:136-48.

85. Kleim JA, Jones TA, Schallert T, Motor enrichment and the induction of plasticity before or after brain injury. Neurochem induction of plasticity

86. Martin LR, Williams SL, Haskard KB, et al., The challenge of patient adherence, Ther Clin Risk Manag, 2005;1:189-99.

87. Meimoun M, Bayle N, Baude $\mathrm{M}$, et al., [Intensity in the neurorehabilitation of spastic paresis], Rev Neurol (Paris), 2015;171:130-40

88. Veerbeek JM, Koolstra M, Ket JC, et al., Effects of augmented exercise therapy on outcome of gait and gait-related activities in the first 6 months after stroke: a meta-analysis, Stroke, 2011;42:3311-15. 


\section{Spastic Paresis and Rehabilitation - The Patient Journey}

David Bowers, ${ }^{1}$ Klemens Fheodoroff, ${ }^{2}$ Patricia Khan, ${ }^{3}$ Julian P Harriss, ${ }^{4}$ Khashayar Dashtipour, ${ }^{5}$ Laxman Bahroo, ${ }^{6}$ Michael Lee, ' Denis Zakharov, ${ }^{8}$ Jovita Balcaitiene ${ }^{9}$ and Virgilio Evidente ${ }^{10}$

1. Siskin Spine and Rehabilitation, Chattanooga, Tennessee, USA; 2. Department of Neurorehabilitation Gailtal-Klinik, Hermagor, Austria; 3. Catarinense Center of Rehabilitation, Florianopolis, Brazil; 4. King's College London and St Thomas' Hospital London, UK; 5. Division of Movement Disorders, Department of Neurology, Loma Linda University School of Medicine, Loma Linda, California, USA; 6. Department of Neurology, Georgetown University Hospital, Washington DC, USA; 7. Department of Physical Medicine and Rehabilitation, School of Medicine, University of North Carolina, Chapel Hill, North Carolina, USA; 8. Scientific Research Psychoneurological Institute, St. Petersburg, Russia; 9. Ipsen Pharma, Paris, France; 10. Movement Disorders Center of Arizona, Scottsdale, USA

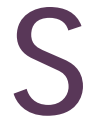
pastic paresis is a complex condition associated with damage to the upper motor neurons, typically caused by cerebral palsy, multiple sclerosis, stroke or trauma. Despite substantial impact on patients' independence and burden on caregivers, there is a lack of consensus on optimal management of this condition and the patient journey remains unclear. A group of physicians, experienced in spasticity management, recently convened with the objective of analysing the patient journey from a care pathway perspective in different geographical regions and under different conditions from acute phase to long-term/chronic disease status. The experts reviewed results from recent patient and healthcare practitioner surveys on the subject and assessed how current patient pathways could be improved, using their own experiences to highlight the issues related to management deficiencies in their individual countries. The group divided the patient journey into steps, considering the evidence from the point of view of healthcare practitioners, patients, caregivers and funders/payors. This paper is a response to the lack of consensus on the optimal management of spastic paresis, and acts as a call to action to develop a consistent care pathway that could be applied across a broad range of illnesses, using an interdisciplinary approach.

\section{Keywords}

spastic paresis, patient rehabilitation, patient journey, patient care, care pathway

Disclosure: David Bowers has participated in an advisory board for Ipsen and has been a trainer for Allergan and Medtronic. Klemens Fheodoroff has received unrestricted research grants from Ipsen and Merz, and honoraria for instructional courses from Ipsen, Allergan and Merz. Patricia Khan has participated in advisory boards for Ipsen, and has received honoraria for instructional courses from Ipsen and Allergan. Julian P Harriss has received research funding into current practice in spasticity management (the SPACE study, supported by Merz Pharma) and expenses for participation in expert panels (Ipsen). Khashayar Dashtipour has received compensation/honoraria for services as a consultant or an advisory committee member or speaker from Allergan, Inc., Ipsen Biopharmaceuticals, Inc., Lundbeck Inc., Merz Pharmaceuticals, Teva Pharmaceutical Industries Ltd., UCB Inc., Impax Pharmaceutical and US World Meds. Laxman Bahroo has received personal compensation from Teva Neuroscience, UCB pharma, Impax, Allergan, Ipsen, US World Meds, AbbVie, Lundbeck and Acadia for consulting, serving on a scientific advisory board or speaking activities, and research support from Ipsen and Teva Neuroscience. Michael Lee has participated in an advisory board for Ipsen. Denis Zakharov has received personal compensation from Ipsen and Merz for consulting, serving on a scientific advisory board or speaking activities. Jovita Balcaitiene is an employee of Ipsen Pharma. Virgilio Evidente has received research support from Ipsen and honoraria for consulting and/or speaking from Ipsen, Merz, Solstice, US WorldMeds, Lundbeck, UCB, Xenoport, Teva, Medtronic and Acadia. This article involves a review of a meeting and did not involve any studies with human or animal subjects performed by any of the authors.

Acknowledgements: The authors thank all of the participants at the meeting and Ipsen Pharma. The authors would also like to thank Adelphi Research for providing assistance with the patient and healthcare practitioner surveys, which were funded by Ipsen Pharma. Medical writing support was provided by Jo-anne Jess, MedSense Ltd., High Wycombe, UK and funded by Ipsen Pharma.

Authorship: All named authors meet the International Committee of Medical Journal Editors (ICMJE) criteria for authorship of this manuscript, take responsibility for the integrity of the work as a whole, and have given final approval to the version to be published.

open Access: This article is published under the Creative Commons Attribution Noncommercial License, which permits any non-commercial use, distribution, adaptation and reproduction provided the original author(s) and source are given appropriate credit.

Received: 22 August 2016

Accepted: 26 October 2016

Citation: European Neurological Review, 2016;11(2):87-95

Corresponding Author: David Bowers, Siskin Spine and Rehabilitation, Chattanooga, Tennessee, USA.

E:dbowers@siskinrehab.org

Support: This paper originates from a report from a meeting on 'The patient journey with spasticity' held in New York, USA, 13 December 2013. This meeting was supported by Ipsen Pharma. The publication of this article was supported by Ipsen Pharma, who were given the opportunity to review the article for scientific accuracy before submission. Any resulting changes were made at the author's/authors' discretion 